\title{
Real-time temperature monitoring with fiber Bragg grating sensor during diffuser-assisted laser-induced interstitial thermotherapy
}

Ngot Thi Pham

Seul Lee Lee

Suhyun Park

Yong Wook Lee

Hyun Wook Kang 


\title{
Real-time temperature monitoring with fiber Bragg grating sensor during diffuser-assisted laser-induced interstitial thermotherapy
}

\author{
Ngot Thi Pham, ${ }^{\text {a }}$ Seul Lee Lee, ${ }^{a}$ Suhyun Park, ${ }^{\mathrm{b}}$ Yong Wook Lee, ${ }^{\mathrm{c}, \mathrm{d}, \star}$ and Hyun Wook Kang ${ }^{\mathrm{d}, \mathrm{e}, *}$ \\ ${ }^{a}$ Pukyong National University, Interdisciplinary Program of Marine-Bio, Electrical and Mechanical Engineering, Busan, Republic of Korea \\ ${ }^{\mathrm{b} C}$ hung-Ang University, School of Electrical and Electronics Engineering, Seoul, Republic of Korea \\ ${ }^{\mathrm{C} P u k y o n g}$ National University, School of Electrical Engineering, Busan, Republic of Korea \\ dPukyong National University, Center for Marine-Integrated Biomedical Technology, Busan, Republic of Korea \\ ePukyong National University, Department of Biomedical Engineering, Busan, Republic of Korea
}

\begin{abstract}
High-sensitivity temperature sensors have been used to validate real-time thermal responses in tissue during photothermal treatment. The objective of the current study was to evaluate the feasible application of a fiber Bragg grating (FBG) sensor for diffuser-assisted laser-induced interstitial thermotherapy (LITT) particularly to treat tubular tissue disease. A $600-\mu \mathrm{m}$ core-diameter diffuser was employed to deliver 980-nm laser light for coagulation treatment. Both a thermocouple and a FBG were comparatively tested to evaluate temperature measurements in ex vivo liver tissue. The degree of tissue denaturation was estimated as a function of irradiation times and quantitatively compared with light distribution as well as temperature development. At the closer distance to a heat source, the thermocouple measured up to $41 \%$ higher maximum temperature than the FBG sensor did after 120 -s irradiation (i.e., $98.7^{\circ} \mathrm{C} \pm 6.1^{\circ} \mathrm{C}$ for $\mathrm{FBG}$ versus $131.0^{\circ} \mathrm{C} \pm 5.1^{\circ} \mathrm{C}$ for thermocouple; $p<0.001)$. Ex vivo porcine urethra tests confirmed the real-time temperature measurements of the FBG sensor as well as consistently circumferential tissue denaturation after 72-s irradiation (coagulation thickness $=2.2 \pm 0.3 \mathrm{~mm}$ ). The implementation of FBG can be a feasible sensing technique to instantaneously monitor the temperature developments during diffuser-assisted LITT for treatment of tubular tissue structure. @ 2017 Society of Photo-Optical Instrumentation Engineers (SPIE) [DOI: 10.1117/1.JBO.22.4.045008]
\end{abstract}

Keywords: fiber Bragg grating temperature sensor; diffusing fiber; laser-induced interstitial thermotherapy; coagulation tissue.

Paper 160832PR received Dec. 6, 2016; accepted for publication Mar. 28, 2017; published online Apr. 20, 2017.

\section{Introduction}

Urethral stricture occurs when a part of the urethral luminal diameter becomes narrowed due to ischemic fibrosis (spongiofibrosis), which manifests as scar tissue in the spongy erectile tissue (corpus spongiosum). ${ }^{1}$ Male urethral stricture accounts for about 5000 inpatient visits and 1.5 million office visits per year in the USA. ${ }^{2}$ The prevalence rate of the urethral stricture has been estimated to be $\sim 0.6 \%$ (i.e., $800 / 100,000$ cases) with $0.2 \%$ in younger men and $0.6 \%$ in older men ( $>65$ years old). ${ }^{1}$ In contemporary practices, most urethral strictures encountered are idiopathic, traumatic, inflammatory, or iatrogenic. ${ }^{2-4}$ The patients with symptomatic stricture disease typically present obstructive voiding symptoms such as straining, incomplete emptying, and a weak stream; they might also have a history of recurrent urinary tract infection, prostatitis, epididymitis, hematuria, or bladder stones. ${ }^{2}$ Depending upon the characteristics of the stricture (i.e., location, etiology, size, and density of spongiofibrosis), a number of invasive treatments have been performed, ${ }^{1}$ including urethroplasty, balloon dilation, cold knife incision, and laser urethrotomy. ${ }^{3-8}$ Currently, the most popular treatment method of urethral strictures is open reconstructive surgery of the urethra (also called urethroplasty). ${ }^{6}$ Yet, in spite of a success rate of $\sim 97 \%$, the procedure is highly invasive, expensive, and related to considerable complications. ${ }^{6}$ The

*Address all correspondence to: Yong Wook Lee, E-mail: yongwook@pknu.ac .kr; Hyun Wook Kang, E-mail: wkang@ pukyong.ac.kr balloon dilation injects a small tube into a urethra to keep the stricture open and stretches fibrotic tissue to widen the urethral lumen. ${ }^{2}$ The cold knife incision releases scar tissue and increases the size of the urethral lumen. ${ }^{5}$ However, these minimally invasive treatment methods have presented high complications such as discomfort and pain and risk of recurrence due to incomplete ablation of the scar tissue. ${ }^{5-7}$ In the case of laser ablation, the delivery of laser light through an end-firing fiber can coagulate or ablate the target tissue for scar removal, but the forward light emission often limits the extent of treatment areas. $^{5-8}$

Laser-induced interstitial thermotherapy (LITT and also called interstitial LA $)^{9}$ has been investigated and used as a minimally invasive method for treating different types of tumors in liver, brain, head, neck, and other tissues in terms of thermal coagulation. ${ }^{10-14}$ LITT delivers laser light through a miniature optical fiber percutaneously to the point of treatment. Volumetric absorption of laser energy within the tissue results in the localized heating, leading to thermal damage to the target tissue in terms of irreversible thermal denaturation. ${ }^{10}$ However, laser light during LITT often results in less uniform volumetric heat deposition due to geometrical variations in the tumors. ${ }^{10,12,15}$ In addition, the eventual goal of LITT is to coagulate or even ablate the entire pathological tissue with no or minimal thermal injury to the surrounding healthy tissue. ${ }^{10,13}$

1083-3668/2017/\$25.00 @ 2017 SPIE 
Thus, to entail more consistent light distribution as well as to real-time monitor temperature developments during laser treatment would be critical for ensuring the safety and efficacy of thermal treatment on soft tissue.

Recently, temperature monitoring during LITT for pathological tissue has been performed with thermal sensors in light of contact techniques [e.g., thermocouples (TCs) ${ }^{16,17}$ and fiber Bragg gratings (FBGs) ${ }^{18,19}$ ] and noncontact techniques (e.g., magnetic resonance imaging ${ }^{20,21}$ ). On account of cost-effectiveness and easy utilization, the contact method has primarily been used for most LITT processes. ${ }^{10,15}$ In fact, TCs are moderately inexpensive, quite accurate $\left(\sim 1^{\circ} \mathrm{C}\right)$, and associated with a relatively short response time (i.e., shorter than $1 \mathrm{~s}$ ). ${ }^{17}$ However, the close placement of TCs to the light source often involves direct light absorption and self-heating, leading to overestimation and rather inaccurate recording of the interstitial tissue temperature. ${ }^{16,17}$ On the other hand, due to high optical sensitivity, FBGs have recently attracted great attention as versatile optical sensing characteristics employed for various biomedical applications such as temperature recording, ${ }^{1,22-25}$ strain estimation, ${ }^{25,26}$ and pressure measurement. ${ }^{26,27}$ Unlike TCs in association with possible light absorption, significantly low light absorption $(<7 \%)$ at near-infrared wavelengths may enable FBGs to overcome the limitation of TCs (i.e., temperature overestimation resulting from self-heating) and to eventually provide precise information on temporal elevations of the tissue temperature during laser treatment. ${ }^{23,28}$ Therefore, the aim of the current study is to identify the feasible application of FBG as a biomedical temperature sensor to monitor in real time the interstitial temperature in urethral tissue during diffuser-assisted LITT. Both an FBG and a TC as temperature sensing elements were quantitatively compared in terms of temperature variations at different positions away from the diffuser. The degree of tissue coagulation was also evaluated with the temperature monitored real time in tissue.

\section{Materials and Methods}

A customized diffusing applicator (Tecure, Busan, Republic of Korea) was fabricated to circumferentially coagulate tissue during diffuser-assisted LITT. The diffusing tip was prepared in a length of $20 \mathrm{~mm}$ and a diameter of $0.6 \mathrm{~mm}$ for delivery of high laser power $(980 \mathrm{~nm})$. For mechanical protection, the tip was covered with a customized glass cap (1.4 mm in outer diameter). Figure 1(a) shows a polar measurement setup to characterize spatial light distribution from the diffuser by using a customized goniometer in conjunction with a photodiode (PD-300-3W, Ophir, Jerusalem, Israel). The photodiode was initially rotated around the proximal end of the diffuser at a radius of $4 \mathrm{~mm}$ with an increment of $10 \mathrm{deg}$ to measure polar emissions. After each round of the measurement, the diffuser was then moved toward the distal end by $1 \mathrm{~mm}$, and another polar measurement continued to estimate the longitudinal emissions along the diffuser axis. As each photodiode had a spectral range of 350 to $1100 \mathrm{~nm}$, the laser light $(980 \mathrm{~nm})$ was applied to evaluate the emission profiles. All the measured intensities were normalized by using the maximum intensity to have the minimal effect of power variations on quantitative evaluations. Figure 1(b) shows a 3-D image of the emission profile from the diffusing tip. In spite of symmetric radial diffusion, the overall longitudinal distribution was skewed to the distal end, and the maximum intensity occurred at $5 \mathrm{~mm}$ away from the proximal end. Figure 1(c) exhibits almost isotropic polar emissions at the maximum intensity with a deviation of less than $10 \%$ (i.e., $0.86 \pm 0.09$ ). The mean polar intensity was estimated to be $0.90 \pm 0.05$. According to power measurements, $89 \%$ and $11 \%$ were transmitted in the radial and forward directions, respectively, (around $10 \%$ insertion loss).

Numerical computations were performed to predict the spatial distribution of the interstitial temperature as well as the degree of thermal denaturation in porcine liver tissue during diffuser-assisted LITT by using COMSOL Multiphysics software (v5.0, COMSOL Inc., Burlington, Massachusetts). The geometry for the simulation used a 20 -mm long diffusing tip (600- $\mu \mathrm{m}$ core diameter) with the distal end glass-capped (length $=21 \mathrm{~mm}$ and outer diameter $=1.4 \mathrm{~mm}$ ) as shown in Fig. 2(b). The size of the liver tissue was $30 \times 30 \times 30 \mathrm{~mm}^{3}$ (tissue boundary), and 4-W 980-nm laser light was cylindrically irradiated through the diffuser for $120 \mathrm{~s}$. Table 1 shows thermal properties of the materials utilized for the current computations.

The thermal response of the tissue during the irradiation was estimated by ${ }^{31}$

$\rho c \frac{\partial T(r, t)}{\partial t}=\nabla \cdot[k \nabla T(r, t)]+Q_{l}$,

where $\rho\left(\mathrm{kg} / \mathrm{m}^{3}\right)$ is the tissue density, $c(\mathrm{~J} / \mathrm{kg} \cdot \mathrm{K})$ is the tissue specific heat, $k(\mathrm{~W} / \mathrm{m} \cdot \mathrm{K})$ is the tissue heat conductivity, and $T$ $\left({ }^{\circ} \mathrm{C}\right)$ is the tissue temperature. The effect of blood perfusion and metabolic heat generation was de facto 0 due to the use of the $e x$ vivo model. ${ }^{29,32} Q_{l}\left(\mathrm{~W} / \mathrm{m}^{3}\right)$ is the heat source induced by laser light. In addition, the preliminary goniometric measurement

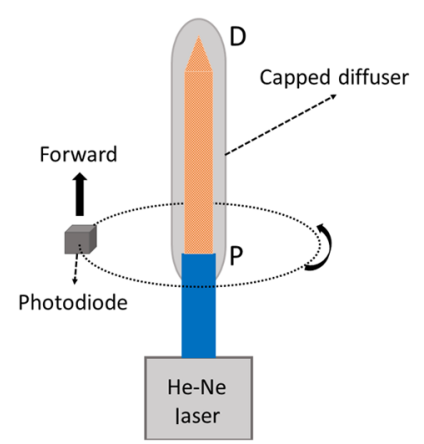

(a)

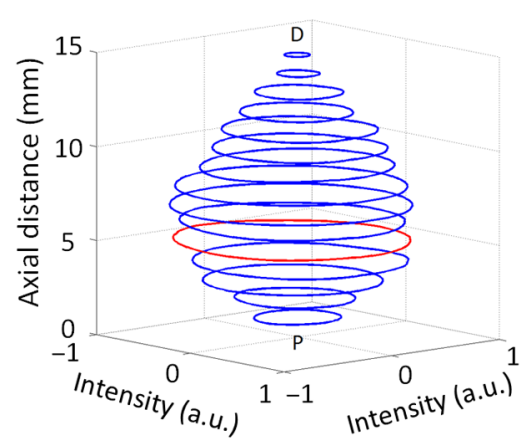

(b)

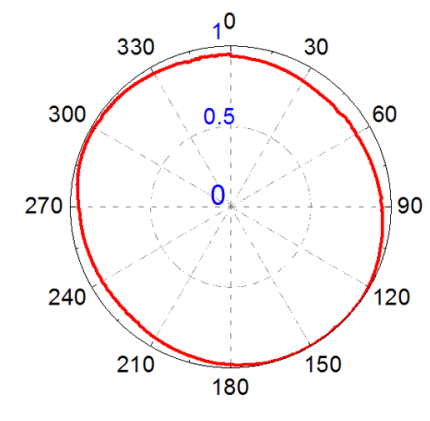

(c)

Fig. 1 Characteristics of diffusing applicator: (a) microscopic image, (b) 3-D emission profile, and (c) polar angle emission at highest intensity (P, proximal; D, distal ends). 


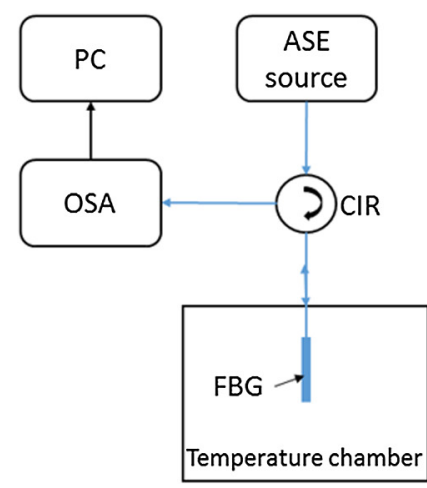

(a)

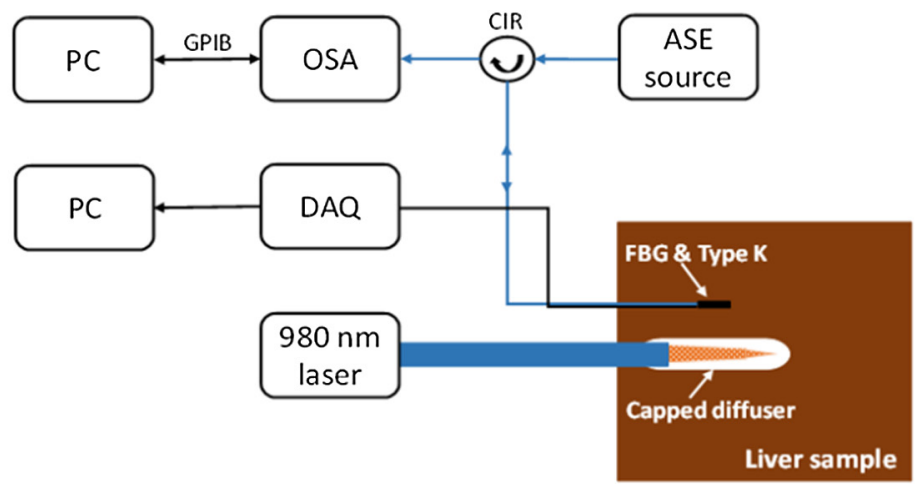

(b)

Fig. 2 Schematic illustration of experimental setups: (a) static calibration of temperature with bare FBG and (b) real-time measurement of temperature in ex vivo bovine liver during diffuser-assisted LITT (ASE, amplified spontaneous emission; OSA, optical spectrum amplifier; CIR, circulator; PC, personal computer; GPIB, general purpose interface bus; DAQ, data acquisition).

confirmed that the laser intensity was delivered in two directions: radially emitted power from the diffusing part $P_{1}(89 \%$ of incident laser power in $\mathrm{W}$ ) and forward emitted power from the fiber tip $P_{2}$ (11\% of incident laser power in $\left.\mathrm{W}\right)$. Thus, the spatial beam intensity in the radial direction was assumed to be cylindrical along the diffuser axis, and the corresponding heat source was expressed as

$Q_{l, \text { radial }}=\mu_{a} \cdot \frac{P_{1}}{2 \pi r l} e^{-\left(\mu_{a}+\mu_{s}\right) r}$,

where $\mu_{a}\left(\mathrm{~cm}^{-1}\right)$ is the absorption coefficient of the tissue, $\mu_{s}$ $\left(\mathrm{cm}^{-1}\right)$ is the scattering coefficient of the tissue, $r(\mathrm{~m})$ is the radial distance from the diffuser surface, and $l(\mathrm{~m})$ is the diffuser length. Based upon the beam divergence from the diffuser tip $(\mathrm{NA}=0.5)$, the beam distribution in the forward direction was assumed to be Gaussian, and the heat source was estimated as

$Q_{l, \text { forward }}=\mu_{a} \frac{P_{2}}{\pi \sigma^{2}} e^{-r^{2} /\left[2 \sigma^{2} \exp \left(\mu_{s} z\right)\right]} \cdot e^{-\left(\mu_{a}+\mu_{s}\right) z}$,

where $\sigma(\mu \mathrm{m})$ is the spot size of the laser beam and $z(\mathrm{~m})$ is the axial depth in tissue. Due to application of the 980-nm wavelength, both the absorption and the scattering effects were significant. ${ }^{30}$ Thus, the effective attenuation coefficient was utilized instead of the absorption coefficient by ${ }^{33}$

$\mu_{\mathrm{eff}}=\sqrt{3 \mu_{a}\left[\mu_{a}+\mu_{s}(1-g)\right]}$.

The initial temperature for the entire tissue was set to be $20^{\circ} \mathrm{C}$, and the external tissue surface (i.e., $30 \times 30 \times 30 \mathrm{~mm}^{3}$ ) was insulated (i.e., $\vec{n} \cdot k \nabla T=0$, where $\vec{n}$ is the direction of the

Table 1 Thermal properties of materials.

\begin{tabular}{lcccc} 
Property & $k(\mathrm{~W} / \mathrm{m} \cdot \mathrm{K})$ & $\rho\left(\mathrm{kg} / \mathrm{m}^{3}\right)$ & $c(\mathrm{~J} / \mathrm{kg} \cdot \mathrm{K})$ & Reference \\
\hline Glass & 1.3 & 2200 & 820 & 29 \\
Air & 0.0257 & 1.205 & 1005 & 29 \\
Liver & 0.513 & 1050 & 2600 & 30 \\
\hline
\end{tabular}

heat flux). Moreover, Arrhenius parameters were utilized to determine the degree of thermal injury primarily due to the temperature dependence of molecular reaction rates. As a first-order rate process, the tissue thermal damage was described by using the Arrhenius damage integral as ${ }^{34}$

$\Omega(r, t)=A_{f} \cdot \int_{0}^{\tau} \exp \left[\frac{-E_{a}}{R T(r, t)}\right] \mathrm{d} t$,

where $\Omega$ is a dimensionless factor to define the irreversible thermal injury, $A_{f}(1 / \mathrm{s})$ is the frequency factor, $E_{a}(\mathrm{~J} / \mathrm{mol})$ is the denaturation activation energy, $R$ is the universal gas constant of $8.314(\mathrm{~J} / \mathrm{mol} \cdot \mathrm{K})$, and $\tau(\mathrm{s})$ is the irradiation time. Thus, the onset of the irreversible thermal denaturation corresponds to the interstitial tissue temperature of $60^{\circ} \mathrm{C}$ to $65^{\circ} \mathrm{C}$, where $\Omega=1 .^{35}$ Table 2 demonstrates the optical properties of the liver under $980 \mathrm{~nm}$, which were assumed to be constant during LITT.

FBG is typically fabricated by irradiating UV light in a wavelength range of 244 to $248 \mathrm{~nm}$ on a Ge-doped photosensitive or hydrogen-loaded single-mode fiber with a spatial pattern, which renders the refractive index of the fiber core be periodically modulated. The periodic core index modulation creates core modes, which are reflected from or transmitted through numerous index boundaries and interfere with each other. In turn, an input beam experiences strong reflection only at the specific wavelength that is determined by a certain phase-matching condition. The reflection wavelength is referred to as the Bragg

Table 2 Physical properties of liver tissue.

\begin{tabular}{lccc} 
Parameter & Symbol, unit & Value & Reference \\
\hline Absorption coefficient & $\mu_{\mathrm{a}}, 1 / \mathrm{cm}$ & 0.64 & 36 \\
Scattering coefficient & $\mu_{\mathrm{s}}, 1 / \mathrm{cm}$ & 47.2 & 36 \\
Frequency factor & $A_{f}, 1 / \mathrm{s}$ & $5.5 \times 10^{41}$ & 35 \\
Activation energy & $E_{a}, \mathrm{~J} / \mathrm{mol}$ & $2.77 \times 10^{5}$ & 35 \\
Universal gas constant & $R, \mathrm{~J} / \mathrm{mol} \cdot \mathrm{K}$ & 8.314 & 35 \\
\hline
\end{tabular}


wavelength $\left(\lambda_{B}\right)$ of FBG, and the phase-matching condition, called the Bragg condition, is given by ${ }^{27}$

$\lambda_{B}=2 \Lambda n_{\mathrm{eff}}$,

where $\Lambda$ and $n_{\text {eff }}$ are the grating period and the effective refractive index of the fundamental core mode at the free space wavelength. ${ }^{37} \lambda_{B}$ is affected by a temperature change through the thermal expansion or contraction of the grating period and the thermo-optic effect (thermally induced change in $n_{\text {eff }}$ ), which enables the use of the FBG as a temperature sensing element. A Bragg wavelength shift $\Delta \lambda_{B}$ induced by a temperature change $\Delta T$ is represented as ${ }^{38}$

$\Delta \lambda_{B}=2\left(\Lambda \frac{\partial n_{\mathrm{eff}}}{\partial T}+n_{\mathrm{eff}} \frac{\partial \Lambda}{\partial T}\right) \Delta T=\lambda_{B 0}\left(\alpha_{n}+\alpha_{\Lambda}\right) \Delta T$,

where $\Delta T=\left(T_{H}-T_{0}\right)$ and $T_{0}$ and $T_{H}$ are the reference and elevated temperatures applied to the FBG, respectively. $\lambda_{B 0}$ is the Bragg wavelength of the FBG at $T_{0}$, and $\alpha_{\Lambda}$ and $\alpha_{n}$ are the thermal expansion and thermo-optic coefficients of a singlemode fiber in which the FBG is inscribed, respectively. On the basis of Eq. (6), the thermal sensitivity $S$ of the FBG is defined as ${ }^{38,39}$

$S=\frac{\Delta \lambda_{B}}{\Delta T}=\lambda_{B 0}\left(\alpha_{\Lambda}+\alpha_{n}\right)$.

With the pre-estimated $S$, the measurement of $\Delta \lambda_{B}$ will thus allow the estimation of $\Delta T$. Although the FBG actually has the cross sensitivity to temperature and strain, it is assumed here that $\lambda_{B}$ is shifted only by temperature change as straininduced $\Delta \lambda_{B}$ is small enough to be ignored. ${ }^{23}$ In the current study, an FBG (SMF-28e-acrylate coating, Technica Optical Component, LLC, Beijing, China) was utilized to monitor in real time the interstitial tissue temperature during laser irradiation. The FBG with a 5-mm active length has a reflectivity of $74.5 \%$ at $\lambda_{B}$ of $1550.03 \mathrm{~nm}$, a $3-\mathrm{dB}$ bandwidth of $0.38 \mathrm{~nm}$, and a side-mode suppression ratio of $28 \mathrm{~dB}$.

Prior to application of an FBG sensor for diffuser-assisted LITT, $S_{\mathrm{FBG}}$ was obtained and calibrated by using the relationship between $\Delta \lambda_{B}$ and $\Delta T$ [based on Eq. (7)]. An experimental static calibration was performed under the controlled environmental temperature as shown in Fig. 2(a), for which the experimental setup consisted of an environmental chamber, an FBG, an amplified spontaneous emission (ASE) source, an optical spectral analyzer (OSA), and an optical circulator. Initially, the FBG was placed in the chamber within which temperature could be controlled in a range from $-40^{\circ} \mathrm{C}$ to $150^{\circ} \mathrm{C}$ (TH-KE025 Environmental Chamber 25L, Jeio Tech. Co. Inc, Daejeon, Korea). Broadband light from the ASE source (ASE-FL7701 SCL-band, FiberLabs Inc., Saitama, Japan) reaches the FBG via the circulator. Then reflected signal of the FBG enters the OSA (AQ6370C, Yokogawa, Tokyo, Japan) via the circulator. The reflected spectrum of the FBG was monitored with the OSA. The calibration testing was executed in a temperature range between $20^{\circ} \mathrm{C}$ and $100^{\circ} \mathrm{C}$, and each temperature interval was $10^{\circ} \mathrm{C}$ and lasted for $5 \mathrm{~h}$.

Both a $K$-type thermocouple and FBG were employed to measure temperature elevations in tissue during diffuser-assisted LITT [Fig. 2(b)]. Porcine liver tissue was used as an ex vivo sample due to easy procurement and storage for LITT. All the samples were acquired from a local slaughter house, and each specimen was prepared with a size of $30 \times 30 \times$ $30 \mathrm{~mm}^{3}$ and stored in saline at room tempeature $\left(20^{\circ} \mathrm{C}\right)$ prior to testing. Any discoloration in the sample during LITT indicated irreversible thermal denaturation in the tissue. Then the diffusing applicator was perpendicularly inserted into the ex vivo tissue (20-mm deep). Both the thermocouple and FBG were also inserted into the predetermined position of the tissue parallel to the diffusing device. Various distances of the positions were tested from 0 (contract) to $3.5 \mathrm{~mm}$. Each tip was positioned in the middle of the diffuser in order to measure the maximum temperature increase. The $K$-type thermocouple (TSL-101, Guageworld, Anyang, Republic of Korea) with an accuracy of $\pm 1.2^{\circ} \mathrm{C}$ was connected to a data acquisition (DAQ) module (OMB-DAQ-55, OMEGA Engineering Inc., California) to acquire in real time and record any temperature variations in the tissue. The reflectance spectrum of the FBG was collected simultaneously by using LabVIEW software and controlling OSA through a general purpose interface bus (GPIB-IEEE 4888, National Instruments, Austin, Texas). As a light source, a 980-nm laser system (60-W Laser Diode, Apollo Instrument, Irvine, California) was employed to deliver optical energy in a continuous-wavelength mode through the diffusing applicator at various power levels $(3,4$, and $5 \mathrm{~W}$ ). The corresponding average irradiances on the tissue surface were estimated to be $\sim 2.4,3.2$, and $4.0 \mathrm{~W} / \mathrm{cm}^{2}$, respectively. The initial tissue temperature was maintained at $20^{\circ} \mathrm{C}$ (i.e., no irradiation), and the total irradiation times were varied from 10 to $120 \mathrm{~s}$ with an increment of $10 \mathrm{~s}$. Each experiment was repeated five times $(N=5)$. The transmission loss of the diffusing applicator was measured by using a goniometer after every five tests, and the diffuser was replaced if the transmission dropped by more than $10 \%$ possibly due to tissue contamination or mechanical scratches on the cap. After irradiation testing, each sample was cross sectioned in longitudinal (along diffuser axis) and transverse (6 $\mathrm{mm}$ away from proximal end) directions. Then each cross section was imaged using a digital camera. The spatial extent of coagulation in the tested tissue was measured by using the Image $\mathbf{J}$ (National Institutes of Health, Bethesda, Maryland). The coagulated regions were defined as discoloration (i.e., tan color) after the laser irradiation.

To investigate the feasibility of temperature measurements with the FBG sensor for urological application, porcine urethral tissue was procured from a local slaughter house and prepared in 4.5-cm length and 2-mm inner diameter. Prior to laser irradiation, a diffusing applicator was placed into the tubular urethra and positioned in the center of the lumen. Then the FBG was inserted parallel to the diffuser and situated on the inner wall of the tissue. Due to the size of the protective cap, the diffuser surface was in contact with the luminal wall. The urethral tissue was irradiated in air for $72 \mathrm{~s}$ at $4 \mathrm{~W} 980 \mathrm{~nm}$ to achieve 2-mm-thick thermal coagulation, based upon the results of the ex vivo liver experiments. Five samples were tested under the same irradiation conditions. After the laser treatment, each tested tissue was stored in $10 \%$ neutral-buffered formalin (VWR International, Westchester, Pennsylvania) for three days and embedded in paraffin for histology preparation. Then the treated region was sectioned into $4-\mu \mathrm{m}$-thick specimens along the urethra every $4 \mathrm{~mm}$ (i.e., total five slides per sample) and stained with hematoxylin and eosin (H\&E). An optical transmission microscope was used to image the prepared slides. The degree of thermal denaturation in the urethral tissue was measured with Image $\mathbf{J}(N=10)$ for quantitative evaluations. For 
statistical analysis, Mann U test as a nonparametric method was performed and $p<0.05$ represented insignificant correlation.

\section{Results}

Figure 3 shows the variation of the reflectance spectrum of the FBG, measured by the OSA at various temperatures in the environmental chamber [Fig. 2(a)] for calibration purposes. According to Fig. 3(a), the static calibration curve of the FBG demonstrated that the peak wavelength (power $=-4.9 \mathrm{~dB}$ ) shifted to the longer wavelengths along with temperature increase (i.e., from $1549.80 \mathrm{~nm}$ at $0^{\circ} \mathrm{C}$ to $1550.71 \mathrm{~nm}$ at $80^{\circ} \mathrm{C}$ ). Figure 3(b) shows a linear relationship between the applied temperature and the wavelength shift $\left(R^{2}=0.99\right)$, indicating that the thermal sensitivity of the measuring system is constant. The thermal sensitivity (= spectral shift/temperature change) of the FBG used in the tests was estimated to be $0.0114 \mathrm{~nm} /{ }^{\circ} \mathrm{C}$.

Figure 4 shows transient responses of temperature in ex vivo bovine liver during 980-nm LITT for $90 \mathrm{~s}$ at various power levels $(3,4$, and $5 \mathrm{~W})$. For the sake of comparison, the interstitial temperature at the diffuser surface was measured by using the FBG and type $K$ thermocouple, as shown in Figs. 4(a) and 4(b), respectively. The initial temperature of the tissue was $20^{\circ} \mathrm{C} \pm 1^{\circ} \mathrm{C}(N=5)$. Regardless of the sensor type, the tissue temperature gradually increased with irradiation time as well as laser power. Given the power of $5 \mathrm{~W}$ for 90 -s irradiation, the tissue temperature measured by using the $\mathrm{FBG}$ went up to $98.9^{\circ} \mathrm{C} \pm 7.4^{\circ} \mathrm{C}$ (i.e., $\Delta T=78.9^{\circ} \mathrm{C} \pm 7.4^{\circ} \mathrm{C}$ ), whereas the thermocouple measured up to $144.7^{\circ} \mathrm{C} \pm 6.7^{\circ} \mathrm{C}$ (i.e., $\Delta T=$ $124.7^{\circ} \mathrm{C} \pm 6.7^{\circ} \mathrm{C}$ ). Figure 5 shows temperature variations as a function of irradiation time measured at various positions in the tissue (i.e., 0, 1.5, and $3.5 \mathrm{~mm}$ away from the diffuser surface) during 120-s irradiation at $4 \mathrm{~W}$. Irrespective of the position, both the FBG and the thermocouple confirmed that the interstitial temperature increased with irradiation time. At the diffuser surface (i.e., $0 \mathrm{~mm}$ as direct contact), the thermocouple measured up to $41.0 \%$ higher temperature and $43.1 \%$ faster transient temperature change than the FBG did after 120-s irradiation (i.e., $98.7^{\circ} \mathrm{C} \pm 6.1^{\circ} \mathrm{C}$ and $0.7 \pm 0.1^{\circ} \mathrm{C} / \mathrm{s}$ for FBG versus $130.7{ }^{\circ} \mathrm{C} \pm 5.1{ }^{\circ} \mathrm{C}$ and $0.9 \pm 0.1{ }^{\circ} \mathrm{C} / \mathrm{s}$ for thermocouple; $p<0.001)$. However, as the distance increased, both the FBG and the thermocouple reached comparable temperature elevations (i.e., $53.3^{\circ} \mathrm{C} \pm 3.0^{\circ} \mathrm{C}$ and $0.3 \pm 0.02^{\circ} \mathrm{C} / \mathrm{s}$ for $\mathrm{FBG}$ versus $54.0^{\circ} \mathrm{C} \pm 3.5^{\circ} \mathrm{C}$ and $0.3 \pm 0.03^{\circ} \mathrm{C} / \mathrm{s}$ for thermocouple at $3.5 \mathrm{~mm}$ with 120 -s irradiation; $p=0.26$ ).

The temporal development of the measured temperature with the FBG was compared with that of the simulated temperature (red dashed line) at various radial positions in Fig. 6(a). Irrespective of the position, both the simulation and the experiment obtained almost similar temperature elevations except for a slight difference in the initial development. At the diffuser

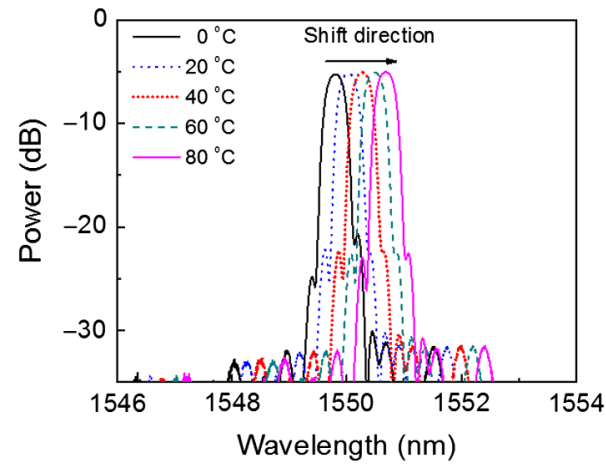

(a)

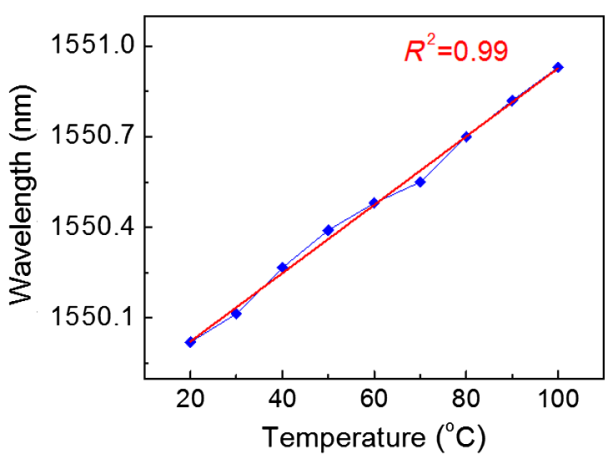

(b)

Fig. 3 Calibrated temperature from bare FBG: (a) variation of FBG reflection spectrum with temperature change and (b) FBG peak wavelength shift as function of temperature.

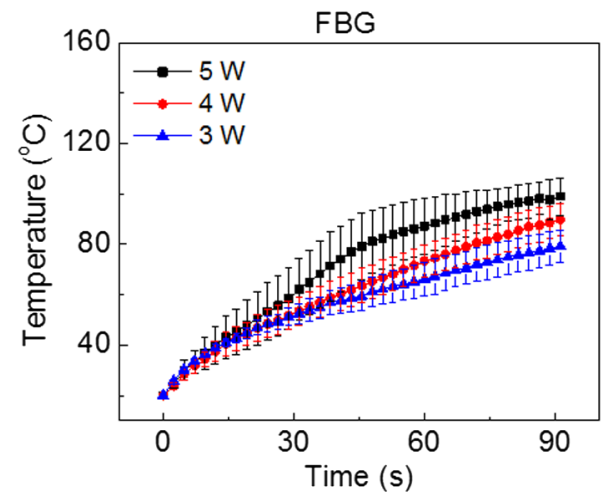

(a)

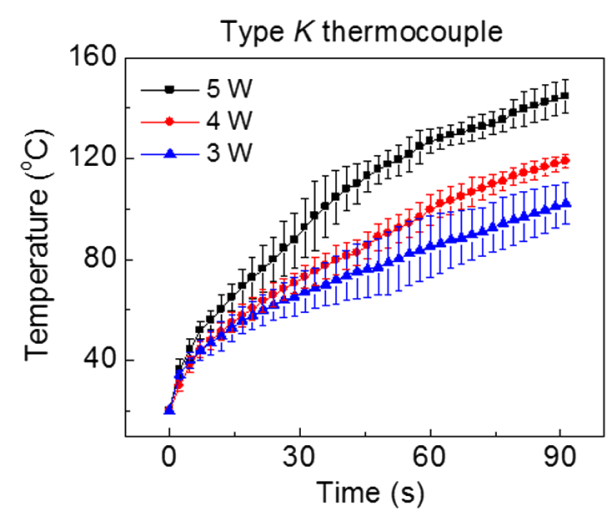

(b)

Fig. 4 Temporal response of temperature in ex vivo bovine liver during 980-nm LITT at various power levels $(3,4$, and $5 \mathrm{~W}$ ) by using: (a) FBG and (b) type $K$ thermocouple positioned at diffuser surface $(N=5)$. 


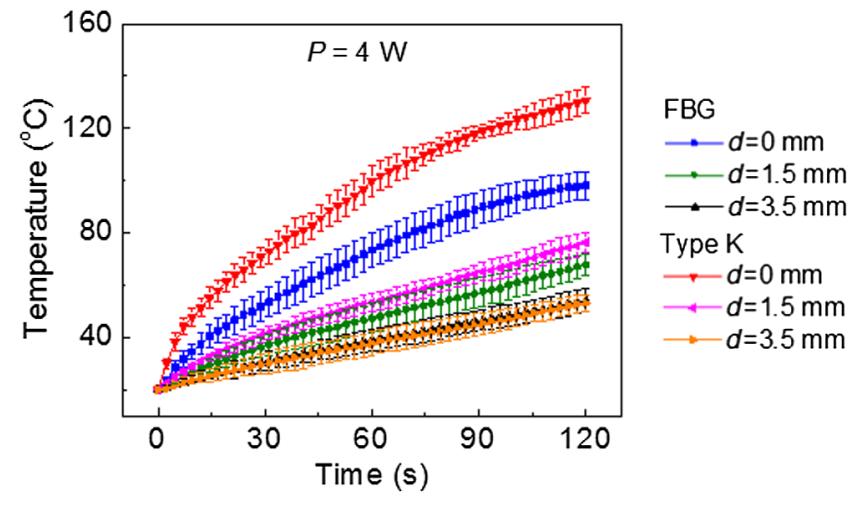

Fig. 5 Comparison of temperature elevations measured from FBG and type $K$ thermocouple at various positions $(0,1.5$, and $3.5 \mathrm{~mm}$ from diffuser surface) during 4-W irradiation for $120 \mathrm{~s}(N=5)$.

surface, the heating rates after $4-\mathrm{W}$ irradiation for $120 \mathrm{~s}$ were estimated to be 0.61 and $0.66 \pm 0.1^{\circ} \mathrm{C} / \mathrm{s}$ for the simulation and the measurement, respectively. Figure 6(b) exhibits the simulated temperature distribution along the diffuser axis after 4-W irradiation for $120 \mathrm{~s}$. The overall distribution was symmetrically cylindrical around the axis, and the temperature decreased significantly with the radial distance. After 120-s irradiation, the temperature at the diffuser surface reached up to 93. $3^{\circ} \mathrm{C}$

Figure 7 shows simulation results of temperature distribution as well as the extent of thermal coagulation in liver tissue after 4$\mathrm{W}$ irradiation for $72 \mathrm{~s}$. The transverse images were acquired from the middle position of the diffuser. According to Fig. 7(a), the temperature developed cylindrically around the diffuser axis (black solid circles), and the maximum temperature $\left(81.5^{\circ} \mathrm{C}\right)$ occurred at the diffuser surface. The dotted line represents the threshold temperature to initiate the irreversible thermal denaturation in the tissue. Figure 7(b) shows the corresponding distribution of the thermal coagulation in tissue. In accordance with the temperature development, the irreversible denaturation (i.e., $\Omega=1$ ) was almost radially formed (black dotted line). The estimated radial coagulation thickness was $1.6 \pm 0.2 \mathrm{~mm}$.

Figure 8 shows thermal responses of bovine liver tissue during 4-W diffuser assisted LITT for 120-s irradiation. According to Fig. 8(a), a series of transverse (left column) and crosssectional (right column) images of the coagulated tissue were captured at various irradiation times ranging from 24 to $120 \mathrm{~s}$. Apparently, the degree of the thermal coagulation (defined as discoloration on tissue surface) evolved with irradiation time. Due to asymmetric beam distribution in Fig. 1(b), the crosssectional images showed coagulative responses in an oval shape. Figure 8(b) compares the temporal development of the interstitial tissue temperature (measured by the FBG was placed at the diffuser surface; black) with that of the measured radial coagulation thickness (blue). The thickness was measured $5 \mathrm{~mm}$ away from the proximal end of the diffuser as the position yielded the maximum intensity [Fig. 1(b)]. Overall, both the temperature and the thickness increased with irradiation time. Unlike the temperature, the extent of the tissue coagulation showed a linear increase $\left(R^{2}=0.99\right)$. In fact, the radial expansion of the tissue denaturation reached up to $3.4 \pm 0.3 \mathrm{~mm}$ (coagulation rate $\approx 0.03 \mathrm{~mm} / \mathrm{s}$; corresponding temperature $=$ $98.7^{\circ} \mathrm{C} \pm 6.1^{\circ} \mathrm{C}$ ) after 120 s. $1.9 \pm 0.3$-mm-thick coagulation was induced at the irradiation time of $72 \mathrm{~s}$, corresponding to $80.1^{\circ} \mathrm{C} \pm 7.2^{\circ} \mathrm{C}$. It was noted that the radial coagulation thickness from the experiments was $\sim 20 \%$ thicker than that from the simulation in Fig. 7(b) i.e., $1.9 \pm 0.3 \mathrm{~mm}$ for tissue versus $1.6 \pm$ $0.2 \mathrm{~mm}$ for model; $p<0.005$ ). Figure $8(\mathrm{c})$ shows the spatial distribution of light intensity (black) and coagulation thickness (blue) along the diffuser axis (i.e., longitudinal distance). Both the intensity and the thickness were normalized for qualitative comparison. According to Fig. 8(c), the overall spatial distributions were quite comparable except for two small humps observed in the measured light intensity. Table 3 summarizes the measured values of temperature and coagulation thickness as shown in Fig. 8(b).

To evaluate urological applications of temperature-monitored diffuser-assisted LITT, porcine urethral tissues were tested with $4 \mathrm{~W} 980 \mathrm{~nm}$ for 72-s irradiation and histologically examined as shown in Fig. 8. The irradiation time was selected to obtain $\sim 2 \mathrm{~mm}$ of thermal coagulation in the urethral tissue. Figure 9(a) shows a gross image of the tested urethra along with cross-sectional (H\&E stained) histology images at various positions (every 4-mm distance) along the diffuser axis. The control (c) shows no tissue deformation (i.e., discoloration), whereas the irradiated regions where the diffuser was positioned (1 to 5) are principally associated with radial tissue coagulation around the lumen. The spatial extent of the denaturation was measured to be $2.2 \pm 0.3 \mathrm{~mm}$, which was slightly thicker $(16 \%)$ than the liver testing under the equivalent conditions [1.9 $\pm 0.3 \mathrm{~mm}$ in Fig. 8(a); $p<0.005]$. It should be noted that the treated tissue induced $\sim 230 \%$ larger luminal area than the control did [Fig. 9(a)]. Furthermore, the middle regions

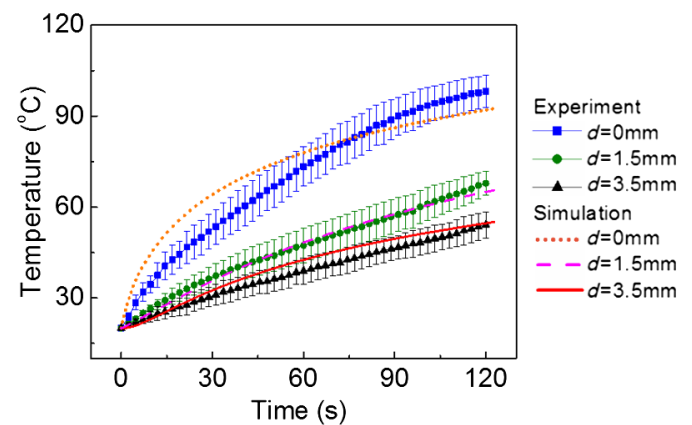

(a)

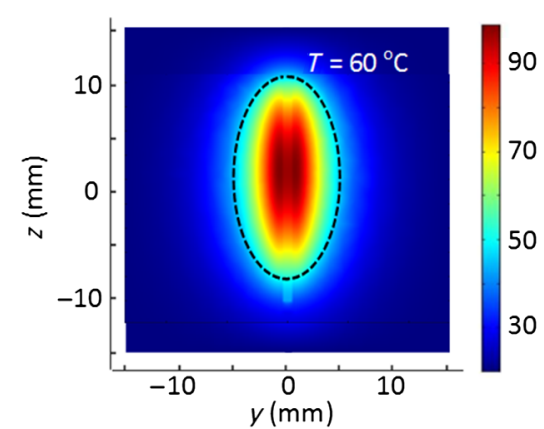

(b)

Fig. 6 Thermal responses of tissue during 4-W irradiation for $120 \mathrm{~s}$ : (a) comparison between simulated and measured temperature at various radial positions $(0,1.5$, and $3.5 \mathrm{~mm}$ from diffuser surface) and (b) simulated temperature distribution along diffuser axis. 


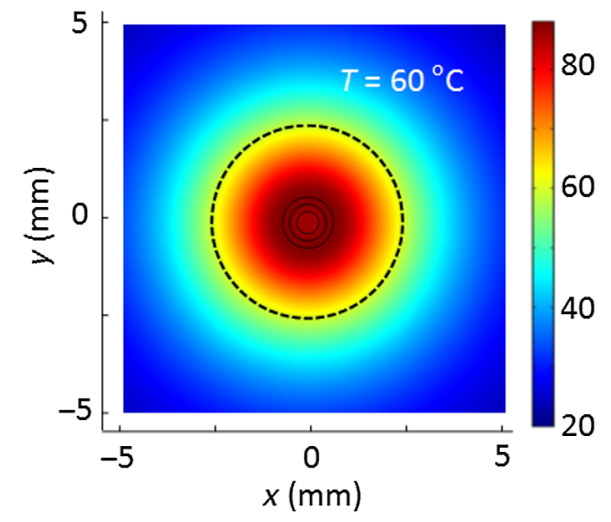

(a)

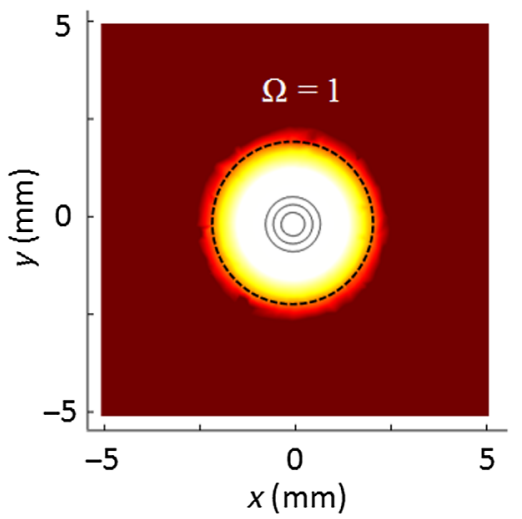

(b)

Fig. 7 Cross-sectional images at middle position of diffusing applicator from numerical simulations (4 W for $72 \mathrm{~s})$ : distribution of (a) temperature and (b) thermal damage $(\Omega=1)$.

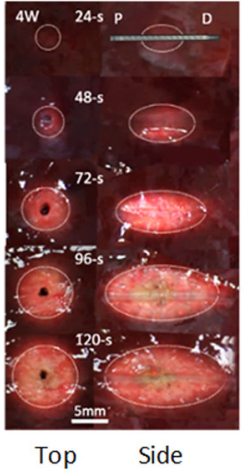

(a)

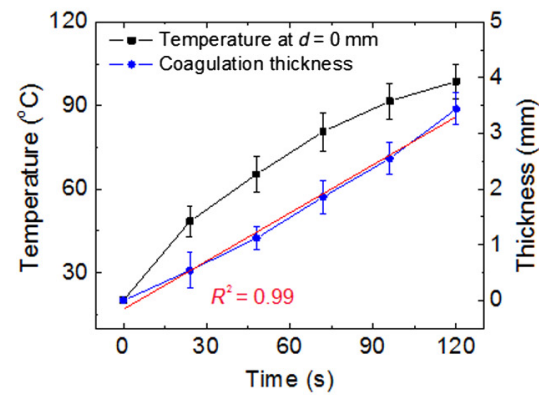

(b)

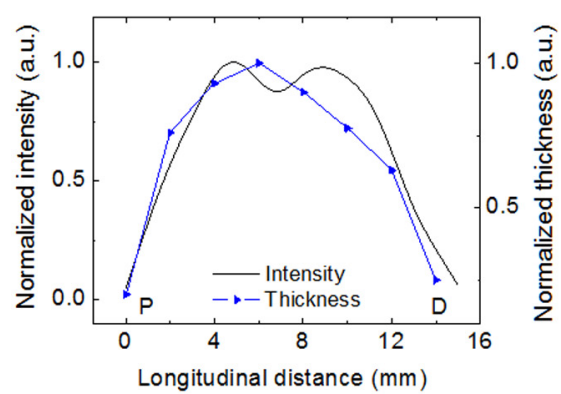

(c)

Fig. 8 Thermal coagulation of ex vivo bovine liver during 4-W diffuser-assisted LITT for $120 \mathrm{~s}$ : (a) compilation of images on irreversible tissue denaturation (left column: top view on transverse view of temperature sections and right column: side view along diffuser axis) at various irradiation times, (b) comparison of FBG-measured temperature at diffuser surface with corresponding transverse coagulation thickness (measured at $5 \mathrm{~mm}$ from proximal end), and (c) quantitative comparison between normalized light intensity and coagulation thickness along diffuser axis (i.e., longitudinal distance) after 120-s photocoagulation at $4 \mathrm{~W}$.

Table 3 Summary of measured temperature and coagulation thickness (mean \pm 1 SD).

\begin{tabular}{|c|c|c|c|c|c|c|}
\hline Time (s) & 0 & 24 & 48 & 72 & 96 & 120 \\
\hline Temperature $\left({ }^{\circ} \mathrm{C}\right)$ & 20 & $48.3 \pm 5.8$ & $65.1 \pm 6.8$ & $80.1 \pm 7.2$ & $91.6 \pm 6.2$ & $98.7 \pm 6.1$ \\
\hline Thickness (mm) & 0 & $0.5 \pm 0.4$ & $1.1 \pm 0.2$ & $1.9 \pm 0.3$ & $2.6 \pm 0.2$ & $3.4 \pm 0.3$ \\
\hline
\end{tabular}

of the irradiated tissue ( 2 and 3 ) created relatively $12.6 \%$ thicker coagulation than the other regions did. Figure 9 (b) shows the magnified images of the control and the tested tissue. Unlike the control, the irreversible coagulation vividly involved epithelial cellular death and formation of vacuolation, resulting in dark, dense, and almost circumferential discoloration around the lumen. Layers of smooth muscle cells with no cellular deformation confirmed native urethral tissue (pinkish color) even after thermal treatment, which was observed in both the control and the treated tissues in Fig. 9(b).
Figure 10(a) shows thermal responses of the urethral tissue during diffuser-assisted LITT. The FBG was located at the center of the diffuser and used to measure temperature variations. Similar to the bovine data in Fig. 8(b), the measured temperature increased with irradiation time. It was noted that the irradiation time of $72 \mathrm{~s}$ resulted in the tissue temperature of $55.4^{\circ} \mathrm{C}$, corresponding to the temperature measured in the bovine tissue in Fig. 8(b). Figure 10(b) compares the longitudinal distribution of the normalized light intensity (black) from the diffuser and the normalized coagulation developed 

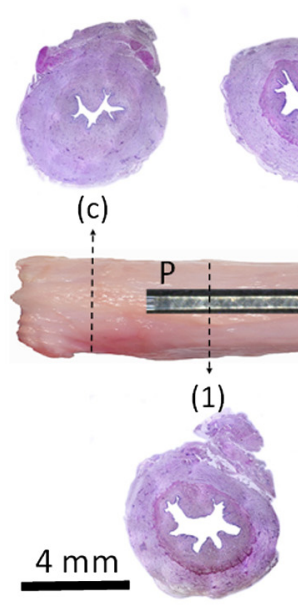

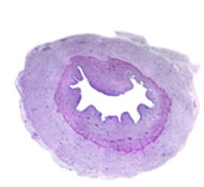

(2)

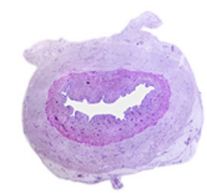

(4)
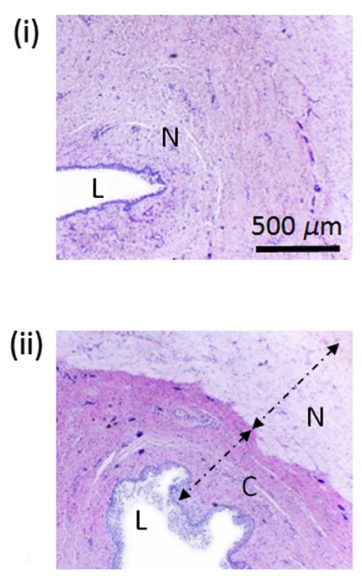

(b)

(a)

(5)

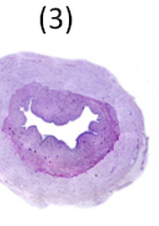

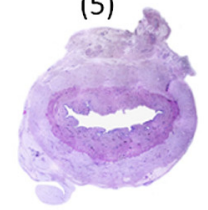

.

Fig. 9 H\&E-stained histology images on porcine urethra after 4-W 980-nm diffuser-assisted LITT for $72 \mathrm{~s}$ : (a) gross and cross-sectional images of urethral tissue [(c), control; (1) to (5), cylindrically coagulated tissue; $\mathrm{P}$, proximal; $\mathrm{D}$, distal ends of diffuser) and (b) magnified (10x) histology image on transition zone [(i) control tissue and (ii) treated tissue from (3) in Fig. 8(a); L, lumen; C, coagulated; N, normal].

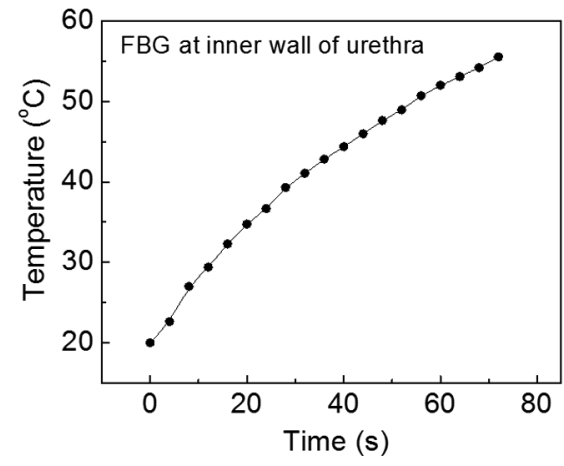

(a)

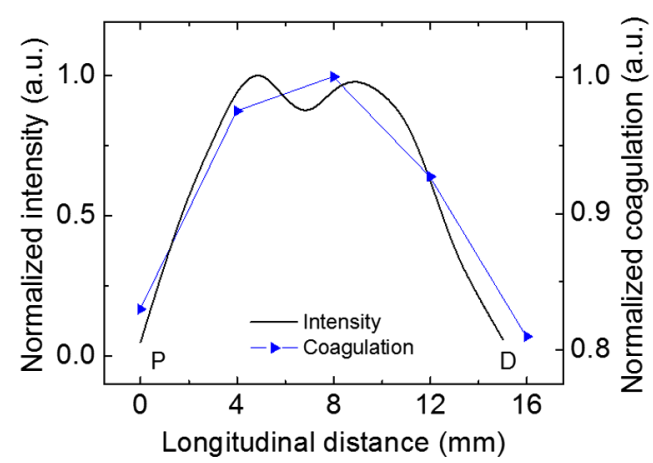

(b)

Fig. 10 Thermal response of ex vivo porcine urethra after 4-W diffuser-assisted LITT for $72 \mathrm{~s}$ : (a) temperature elevations at urethral lumen as function of irradiation time and (b) comparison between normalized light intensity and coagulation thickness along diffuser axis (i.e., longitudinal distance; P, proximal; $D$, distal ends of diffuser).

in a radial direction (blue). The quantitative evaluations confirmed that the radial coagulation patterns agreed well with the spatial light distribution along the axis of the urethra in the current study.

\section{Discussion}

The aim of the current study was to evaluate the feasibility and effectiveness of diffuser-assisted LITT on urethra by assessing and correlating temperature and the induced thermal damage. According to Fig. 5, both the FBG and the thermocouple showed a comparable temperature response when they were positioned relatively far away from the diffuser surface (i.e., maximum measured temperature $=53.3^{\circ} \mathrm{C} \pm 3.0^{\circ} \mathrm{C}$ for $\mathrm{FBG}$ and $54.0^{\circ} \mathrm{C} \pm 3.5^{\circ} \mathrm{C}$ for thermocouple at $3.5 \mathrm{~mm} ; p=0.65$ ). However, as the distance became closer $(0$ and $1.5 \mathrm{~mm})$, the thermocouple measured a temperature increase of up to $\sim 50 \%$ higher than the $\mathrm{FBG}$ did (i.e., $\Delta T=78.9^{\circ} \mathrm{C} \pm 7.4^{\circ} \mathrm{C}$ for $\mathrm{FBG}$ versus $124.7^{\circ} \mathrm{C} \pm 6.7^{\circ} \mathrm{C}$ for thermocouple at $0 \mathrm{~mm}$; Fig. 5). On the other hand, both the simulated and the measured temperatures achieved almost similar elevations at various radial positions [Fig. 6(a)]. All the treated tissue samples vividly experienced discoloration and a dehydrated surface due to irreversible thermal denaturation (Fig. 8), which typically occurs at the tissue temperature of less than $100^{\circ} \mathrm{C} .^{37}$ The identified temperature elevation (i.e., overestimation) could be associated with self-heating due to direct light absorption by the metal tip of the thermocouple. ${ }^{16,17}$ In fact, previous studies showed that the TCs were typically placed $\geq 2 \mathrm{~mm}$ away from a laser-induced heat source to avoid any overestimation of the target temperature. ${ }^{24,40}$ On account of optical fibers with fairly low absorption at the irradiation wavelength $(980 \mathrm{~nm})$, the temperature measurements with the FBG would thereby be more position-independent as well as reliable. Furthermore, thermal coagulation threshold mainly takes place around $60^{\circ} \mathrm{C}$ in soft tissue..$^{15,40}$ According to the current study, the coagulation of liver tissue commenced at the diffuser surface $24 \mathrm{~s}$ after laser irradiation (corresponding temperature $=48.3^{\circ} \mathrm{C} \pm 5.8^{\circ} \mathrm{C}$; Fig. 8). Lower homogeneous thermal gradients from the diffuser could be responsible for the onset temperature lower than the coagulation threshold $\left(60^{\circ} \mathrm{C}\right)$. It should be noted that the active length of the FBG (5 mm) was longer than the axial coagulation depth (i.e., $\sim 2 \mathrm{~mm}$ at 24-s irradiation), which led to fewer precise 
measurements due to inconsistent thermal gradients along the FBG. However, once the coagulation depth was developed up to $6 \mathrm{~mm} 48 \mathrm{~s}$ after the irradiation to cover the entire length of the FBG, the measured temperature exceeded the coagulation temperature (i.e., $65.1^{\circ} \mathrm{C} \pm 6.8^{\circ} \mathrm{C}$ ). Therefore, the FBG could provide more precise information on the interstitial tissue temperature even at the diffuser surface.

According to Figs. 8(b) and 9, the coagulation thickness of urethral tissue was slightly thicker $(16 \%)$ than that of liver tissue under the same experimental conditions (i.e., $2.2 \pm 0.3 \mathrm{~mm}$ for urethra and $1.9 \pm 0.3 \mathrm{~mm}$ for liver; $p=0.15$ ), possibly resulting from different optical properties of the tissues. Absorption of laser energy is primarily followed by thermal coagulation, and the degree of light absorption in tissue is dependent on the wavelength of light and the optical properties of the tissue. ${ }^{41,42}$ As both tissues had similar thermal diffusivities (i.e., $\alpha_{\text {urethra }}=1.4 \times 10^{-7} \mathrm{~m}^{2} / \mathrm{s}$ versus $\left.\alpha_{\text {liver }}=1.3 \times 10^{-7} \mathrm{~m}^{2} / \mathrm{s}\right),{ }^{43,44}$ the extent of the thermal coagulation could primarily be determined by the degree of light absorption, which could explain a further increase of the coagulation thickness in the urethra. However, due to unavailable data, the absorption coefficient of the urethra at $980 \mathrm{~nm}$ should be measured for comparative validation.

Circumferential coagulation was vividly observed in both liver and urethral tissues mainly due to consistent radial emission as shown in Fig. 1(b). Unlike the liver tissue with rather homogeneous glandular tissue structure and circular coagulation [Fig. 8(a)], the urethral tissue exhibited a eccentricity of $1.1 \mathrm{~mm}$ in the denatured area as the inherent tissue structure was less circular with weak integrity (Fig. 9). In fact, the heterogeneous urethral tissue consisted of multilayers such as epithelium, submucosa, and subcutaneous layers. ${ }^{45}$ Furthermore, the out-ofcenter phenomenon could result from the blind placement of the diffusing applicator during the tests. As the lumen of the tissue was in an irregular shape, the diffusing device was difficult to be situated at the center of the urethra, leading to inconsistent tissue contact to ensure complete thermal coagulation. In spite of uniform radial emissions, longitudinal emissions [Fig. 1(b)] contributed variations in the coagulation thickness along the diffuser for both tissue samples [Figs. 8(c) and 9(b)]. According to the normalized coagulation thickness, the middle position of the diffusing applicator entailed more tissue deformation than the proximal and the distal ends, reflecting the spatial distribution of the light emission ( $\sim 10 \%$ deviation). The two peaks in the normalized intensities could chiefly attribute to contribute to the interstitial temperature increase in the tissue, eventually maximizing the temperature between the peaks after conductive heat transfer and yielding the thickest tissue denaturation. It should be noted that the urethral lumen after LITT became up to $230 \%$ larger than the lumen of the control (Fig. 9). In fact, the eventual goal of LITT on urethral stricture is to expand the narrowed urethral pathway for easy and complete urination without development of any complications by circumscribing the thermal coagulation within the wall of the urethra. Thus, the radial dilation of the LITT-treated tissue could be a feasible treatment for tubular tissue with stenosis or stricture. Further in vivo studies will be performed in a mini pig model to validate acute and chronic responses of the urethral tissue after LITT application in terms of hyperemia, healing response, fibrosis, and stricture recurrence. The integration of an inflatable catheter with the proposed diffuserbased LITT is also under consideration to achieve reliable and concentric deployment of the device for interstitial photothermal treatment.

In spite of the feasible application of FBG to estimate the interstitial tissue temperature during photothermal treatment, experimental limitations still remain in translation of the proposed sensing technique into clinical applications. Currently, the simplest method to measure the wavelength-encoded signals of the FBG is to utilize a broadband light source and a spectrum monitoring instrument such as an ASE source and OSA, respectively. However, although the FBG is relatively inexpensive, a spectrum analyzer or an interrogator is quite costly; for example, a commercially available OSA with the minimum resolution bandwidth of $0.01 \mathrm{~nm}$ costs around $\$ 40,000$. As cost-effective approaches, different demodulation techniques have been suggested including ones based on a Fabry-Perot (FP) tunable filter or an edge filter. A fiber FP tunable filter can be used for FBG sensor interrogation by electronically adjusting the distance between two mirrors to make the resonance frequencies shift. A spectral shift of a Bragg resonance can be converted into a temporal shift of an electrical signal in a photodetector following the filter. The demodulation scheme based on the edge filter harnesses its linear power reflectance. That is, a wavelength shift of a Bragg resonance can be obtained by measuring a change in the output power of the edge filter. ${ }^{46}$ Although our experiments were performed by using the OSA, the abovementioned demodulation techniques based on FP tunable filters or edge filters can also be exploited to the currently proposed sensor system for reducing the overall system cost. In addition, the minimum resolution bandwidth of the OSA used in the current study was $0.01 \mathrm{~nm}$, resulting in a thermal sensitivity of $\sim 0.0114 \mathrm{~nm} /{ }^{\circ} \mathrm{C}$ for the current FBG sensor. In turn, the temperature resolution of the sensor was evaluated to be $\sim 0.88^{\circ} \mathrm{C}$, which outperformed that of the thermocouple $\left(\sim 1^{\circ} \mathrm{C}\right)$. Based upon the spatial emission profile of the diffusing tip [Fig. 1(b)], a 5-mm active length of FBG with a reflectivity of $74.5 \%$ was utilized to cover the entire range of thermal gradients in the tissue. However, due to a large coupling constant in comparison with the active length, the used FBG had a large bandwidth of $\sim 0.38 \mathrm{~nm}$, which was difficult to evidently differentiate the acquired FBG peaks. Thus, the coupling constant and the active length of the FBG need to be optimized in order to achieve even a smaller bandwidth for high resolutions. On the other hand, a $\pi$-phase shifted FBG, which provides a much narrower reflection bandwidth $(<0.1 \mathrm{~nm})$, may be an alternative. Additionally, the current study used a single fiber to measure temperature variations at a single point in the tissue, which hardly reflected the overall temperature distribution along the diffuser. In order to evaluate thermal responses at various locations in the tissue lumen, an FBG array (i.e., 1 sensor/cm) can be incorporated into LITT. $^{23}$

\section{Conclusion}

This study demonstrated the feasibility of a single FBG for realtime temperature monitoring during diffuser-assisted photothermal treatment on tubular tissue. Due to high thermal sensitivity and low absorption of irradiation light, the FBG sensor was able to precisely estimate the interstitial tissue temperature in comparison with a thermocouple. Further studies will be performed in vivo to optimize the FBG and its demodulation system to improve the transient response of the temperature during diffuser-assisted laser treatment. 


\section{Disclosures}

The authors declare no conflicts of interest or financial relationships to disclose.

\section{Acknowledgments}

This research was supported by a grant from the Marine Biotechnology Program (20150220) funded by the Ministry of Oceans and Fisheries, Republic of Korea.

\section{References}

1. R. Mathur et al., "A retrospective analysis of urethral strictures and their management at a tertiary care center,' J. Nephro-Urol. Mon. 3(2), 109-113 (2011).

2. L. A. Hampson, J. W. Mcaninch, and B. N. Breyer, "Male urethral strictures and their management," Nat. Rev. Urol. 11(1), 43-50 (2014).

3. A. R. Mundy and D. E. Andrich, "Urethral strictures," BJU Int. 107(1), 6-26 (2011).

4. J. C. Buckley et al., "SIU/ICUD consultation on urethral strictures: dilation, internal urethrotomy, and stenting of male anterior urethral strictures," Urology 83(3), S18-S22 (2014).

5. T. Jin et al., "Safety and efficacy of laser and cold knife urethrotomy for urethral stricture," Chin. Med. J. 123(12), 1589-1595 (2010).

6. N. Fried and D. Ph, "Erbium: YAG laser incision of urethral strictures for treatment of urinary incontinence after prostate cancer surgery," Transforming Healthcare through Innovative and Impactful Research DAMD17-03-1-0087 (2006).

7. G. G. Ferguson et al., "Minimally invasive methods for bulbar urethral strictures: a survey of members of the American Urological Association," Urology 78(3), 701-706 (2011).

8. A. G. Cavalcanti and G. Fiedler, "Opinion: endoscopic urethrotomy," Int. Braz. J. Urol. 41(4), 619-622 (2015).

9. S. N. Goldberg et al., "Image-guided tumor ablation: standardization of terminology and reporting criteria," Radiol. Soc. North Am. 235(3), 728-739 (2012)

10. B. Mensel, C. Weigel, and N. Hosten, "Laser-induced thermotherapy," Recent Results Cancer Res. 167, 69-75 (2006).

11. K. S. Lehmann et al., "In vivo validation of a therapy planning system for laser-induced thermotherapy (LITT) of liver malignancies," Int. J. Colorectal Dis. 26(6), 799-808 (2011).

12. J. W. Rolf Kiessling and O. Winqvist "Laser-induced interstitial thermal therapy for liver metastases," Health Technol. Assess. 2011-0, 1-4 (2011).

13. P. Saccomandi et al., "Theoretical analysis and experimental evaluation of laser-induced interstitial thermotherapy in ex vivo porcine pancreas," IEEE Trans. Biomed. Eng. 59(10), 2958-2964 (2012).

14. M.-F. Marqa et al., "Focal laser ablation of prostate cancer: numerical simulation of temperature and damage distribution," Biomed. Eng. Online 10(1), 45 (2011).

15. T. H. Nguyen et al., "Circumferential irradiation for interstitial coagulation of urethral stricture," Opt. Express 23(16), 20829 (2015).

16. F. Manns et al., "In situ temperature measurements with thermocouple probes during laser interstitial thermotherapy (LITT): quantification and correction of a measurement artifact," Lasers Surg. Med. 23(2), 94-103 (1998).

17. S. A. van Nimwegen et al., "Nd:YAG surgical laser effects in canine prostate tissue: temperature and damage distribution," Phys. Med. Biol. 54(1), 29-44 (2009).

18. P. Roriz et al., "Review of fiber-optic pressure sensors for biomedical and biomechanical applications," J. Biomed. Opt. 18(5), 050903 (2013).

19. E. Schena et al., "Fiber optic sensors for temperature monitoring during thermal treatments: an overview," Sensors 16(8), 1144 (2016).

20. F. A. Jolesz, G. P. Zientara, and D. Ph, "MRI-guided laser-induced interstitial thermotherapy: basic principles," SPIE Institute on Laser-Induced Interstitial Thermotherapy, pp. 294-324 (1995).

21. R. Medvid et al., "Current applications of MRI-guided laser interstitial thermal therapy in the treatment of brain neoplasms and epilepsy: a radiologic and neurosurgical overview," Am. J. Neuroradiol. 36(11), 1998-2006 (2015).
22. E. Schena and L. Majocchi, "Assessment of temperature measurement error and its correction during Nd:YAG laser ablation in porcine pancreas," Int. J. Hyperthermia 30(5), 328-334 (2014).

23. D. Tosi, E. G. Macchi, and A. Cigada, "Fiber-optic temperature and pressure sensors applied to radiofrequency thermal ablation in liver phantom: methodology and experimental measurements," J. Sens. 2015, 1-22 (2015).

24. W. Chen et al., "Performance assessment of FBG temperature sensors for laser ablation of tumors," in IEEE Int. Symp. on Medical Measurements and Applications (MeMeA '15) (2015).

25. L. Dziuda et al., "Fiber Bragg grating-based sensor for monitoring respiration and heart activity during magnetic resonance imaging examinations," J. Biomed. Opt. 18(5), 057006 (2013).

26. H. J. Kalinowski et al., "Application of fibre Bragg grating sensors in biomechanics," Trends Photonics 661(2), 315-343 (2010).

27. S. Poeggel et al., "Optical fibre pressure sensors in medical applications," Sensors 15(7), 17115 (2015).

28. P. Saccomandi et al., "Temperature monitoring and lesion volume estimation during double-applicator laser-induced thermotherapy in ex vivo swine pancreas: a preliminary study," Lasers Med. Sci. 29(2), 607-614 (2014).

29. K. J. Bergemann, R. Krasny, and S. R. Forrest, "Thermal properties of organic light-emitting diodes," Org. Electron. 13(9), 1565-1568 (2012).

30. G. J. Müller and A. Roggan, Laser-Induced Interstitial Thermotherapy, SPIE Press, Bellingham (1995).

31. K. Hashimoto et al., "Permanent hair removal with a diode-pumped Nd: YAG laser: a pilot study using the direct insertion method," J. Am. Acad. Dermatol. 49(6), 1071-1080 (2003).

32. J. Kwon et al., "Computational analysis of endometrial photocoagulation with diffusing optical device," Biomed. Opt. Express 4(11), 2450-2462 (2013)

33. A. Ishimaru, "Diffusion of light in turbid material," Appl. Opt. 28(12), 2210-2215 (1989).

34. S. Thomsen and J. A. Pearce, "Thermal damage and rate processes in biologic tissues," in Optical-Thermal Response of Laser-Irradiated Tissue, A. J. Welch and M. J. C. van Gemert, Eds., pp. 487-549, Springer, Dordrecht, Netherlands (2011).

35. B. Cox, "Introduction to laser-tissue interactions," Opt. Med. PHAS 4886, 1-61 (2007).

36. J. P. Ritz et al., "Optical properties of native and coagulated porcine liver tissue between 400 and 2400 nm," Lasers Surg. Med. 29(3), 205-212 (2001).

37. P. Mégret and C. Caucheteur, "Fiber Bragg gratings: fundamentals and applications," Dr. Sch., pp. 1-31 (2007).

38. Y.-J. Rao, "Fiber Bragg grating sensors: principles and applications," in Optical Fiber Sensor Technology: Devices and Technology, K. T. V. Grattan and B. T. Meggitt, Eds., pp. 355-379, Springer, Boston (1998).

39. A. D. Kersey et al., "Fiber grating sensors," J. Lightwave Technol. 15(8), 1442-1463 (1997).

40. C. Raulin and S. Karsai, Laser and IPL Technology in Dermatology and Aesthetic Medicine, Springer Verlag, Berlin (2011).

41. S. L. Jacques, "Optical properties of biological tissues: a review," Phys. Med. Biol. 58(11), R37-R61 (2013).

42. R. R. Van Den Bos et al., "Endovenous simulated laser experiments at $940 \mathrm{~nm}$ and $1470 \mathrm{~nm}$ suggest wavelength-independent temperature profiles," Eur. J. Vasc. Endovasc. Surg. 44(1), 77-81 (2012).

43. J. A. Balderas-López, A. Mandelis, and J. A. Garcia, "Thermal-wave resonator cavity design and measurements of the thermal diffusivity of liquids," Rev. Sci. Instrum. 71(7), 2933-2937 (2000).

44. J. W. Valvano, J. R. Cochran, and K. R. Diller, "Thermal conductivity and diffusivity of biomaterials measured with self-heated thermistors," Int. J. Thermophys. 6(3), 301-311 (1985).

45. H. Masumoto et al., "Reappraisal of intergender differences in the urethral striated sphincter explains why a completely circular arrangement is difficult in females: a histological study using human fetuses," Anat. Cell Biol. 45(2), 79-85 (2012).

46. Y. Zhao and Y. Liao, "Discrimination methods and demodulation techniques for fiber Bragg grating sensors," Opt. Lasers Eng. 41(1), 1-18 (2004).

Biographies for the authors are not available. 